\title{
Studies on the Interaction between Three Small Flavonoid Molecules and Bovine Lactoferrin
}

\author{
Junyi Huang $\mathbb{D}^{1,2}$ Zhenmin Liu ${ }^{1},{ }^{1}$ Qiaorong $\mathrm{Ma}^{2,3}$ Ziyu He, ${ }^{2}$ Zhidian Niu, ${ }^{2}$ \\ Mengying Zhang, ${ }^{2}$ Liu Pan, ${ }^{2}$ Xiaosheng Qu $\mathbb{D}^{4},{ }^{4}$ Jun Yu $\left(\mathbb{D},{ }^{5}\right.$ and Bing Niu ${ }^{2}{ }^{2}$ \\ ${ }^{1}$ State Key Laboratory of Dairy Biotechnology, Bright Dairy \& Food Co. Ltd., Shanghai, 200436, China \\ ${ }^{2}$ Shanghai Key Laboratory of Bio-Energy Crops, School of Life Sciences, Shanghai University, 200444, China \\ ${ }^{3}$ Clinical Laboratory, Affiliated Minzu Hospital of Guangxi Medical University, Nanning, 530001, China \\ ${ }^{4}$ National Engineering Laboratory of Southwest Endangered Medicinal Resources Development, \\ Guangxi Botanical Garden of Medicinal Plants, 530023 Nanning, China \\ ${ }^{5}$ Department of Ophthalmology, Xinhua Hospital, Shanghai Jiao Tong University School of Medicine, 200092, China
}

Correspondence should be addressed to Xiaosheng Qu; quxs@gxyyzwy.com, Jun Yu; yujun@xinhuamed.com.cn, and Bing Niu; bingniu@shu.edu.cn

Received 16 January 2018; Accepted 13 August 2018; Published 25 September 2018

Academic Editor: Rituraj Purohit

Copyright (C) 2018 Junyi Huang et al. This is an open access article distributed under the Creative Commons Attribution License, which permits unrestricted use, distribution, and reproduction in any medium, provided the original work is properly cited.

\begin{abstract}
The interaction between three flavonoids, i.e., Luteolin (LTL), Quercetin (QCT), and Naringenin (NGN) and bovine lactoferrin (BLF) at pH 7.4 was investigated by fluorescence quenching spectra, synchronous fluorescence spectra, and UV-visible absorption spectra. The results indicate the fluorescence of BLF quenched by Luteolin (LTL), Quercetin (QCT), and Naringenin (NGN) via static quenching. The main force between QCT and LTL with BLF was van der Waals interactions and hydrogen bonds. Electrostatic interactions played a major role in the binding process of interaction between NGN and BLF. Synchronous fluorescence was used to study the conformational changes of BLF. The values of binding constant (Ka) and number of binding sites (n) at different temperatures $(300 \mathrm{~K}, 305 \mathrm{~K}, 310 \mathrm{~K})$ were also calculated, respectively. The results of corresponding thermodynamic parameters as well as binding distance between BLF and LTL, QCT, or GNG were obtained. These results implied that Luteolin (LTL), Quercetin (QCT), and Naringenin (NGN) could provide important guides for compound quantity (e.g., medicine dosage) and the design of new compounds (or drugs).
\end{abstract}

\section{Introduction}

Flavonoids are phytochemicals found in fruit, vegetables, nuts, seeds, stems, and flowers as well as tea, wine, propolis, and honey, which are known to be responsible for colors of many flowers and fruits and protect the plants against pathogens, insects, and UV B radiation [1]. The widespread distribution of flavonoids means that many animals, including humans, ingest significant quantities of flavonoids in their diet. Flavonoids possess many useful properties, including anti-inflammatory activity, oestrogenic activity, enzyme inhibition, antimicrobial activity [2], antiallergenic activity, antioxidant activity, vascular activity, and cytotoxic and antitumor activity [3]. Increasingly, flavonoids are the subject of medical research.
Lactoferrin (LF) is a nonhemic iron-binding glycoprotein found in secretions from exocrine glands, including tears, saliva, semen, bile, and specifically granules of neutrophils [4]. LF is a multifunctional protein involved in many physiological functions, including anti-inflammatory activity, iron transport function, broad-spectrum antibacterial action, regulation of cellular growth and differentiation, and anticancer effect. LF is mainly secreted by the lacrimal gland tear, a small part from the accessory lacrimal gland. The rich content of LF is found in tears, and tear lysozyme, prealbumin, a small amount of secretory immunoglobulin, and growth factors constitute the main ingredients in tear protein. LF is the main defense force to protect the eye from invasion which can cause various ocular surface diseases. In view of its important physiological functions, many drug molecules, 
such as lomefloxacin [5], tosufloxacin [6], oleic acid [7], and polyphenon [8], have been reported to interact with LF.

Protein binding played a potential role in distribution, excretion, and therapeutic effects, and it has been considered as one of the most important physical and chemical characteristics of drugs. The study of the binding of small molecules to protein is an essential and fundamental importance [9]. A new trend in protein-small molecular interaction research is the use of different spectroscopic analyses combined with computational methods (molecular docking) to obtain the mode of interaction of the binding partners [10-13]. Isothermal titration calorimetry (ITC) was also performed [9]. Anbazhagan investigated differential interactions of artemisinin and its derivatives with serum albumin by fluorescence measurements, stopped-flow spectroscopy, and molecular modeling [14]. The UV-visible absorbance spectroscopy and infrared spectroscopy (IR) have been used to research the binding properties of isomeric drugs to BLF [15]. Circular dichroism spectra (CD), a precise method to confirm the changes of proteins conformation, yield information about the three-dimensional structure of protein binding site reduce by small molecules or drugs $[16,17]$. Besides the above methods, Moosavi applied cyclic voltammetric for the molten globule states of cytochrome $\mathrm{c}$ induced by n-alkyl sulfates [18].

As a bioactive compound with antibacterial and antioxidant activities, flavonoid micromolecules may possibly have an impact on activity of BLF in inflammatory reactions. Zhang et al. [19] study the biological implication of the interaction between fibrinogen and resveratrol and observed that fibrinogen strengthened the stability of resveratrol. Li [20] studied interactions between 3 flavonoid compounds and $\alpha$ amylase, and the results showed that the 3 flavonoid compounds are effective inhibitors of $\alpha$-amylase. Therefore, in our study, the interaction between Luteolin (LTL), Quercetin (QCT), Naringenin (NGN), and BLF was investigated with UV-visible spectroscopy and fluorescence spectroscopy.

\section{Materials and Methods}

2.1. Samples and Reagents. Luteolin (LTL), Quercetin (QCT), and Naringenin (NGN) were purchased from Sigma-Aldrich Chemical Co. Ltd. Bovine LF (98\%, purity) was purchased from the Shanghai Huicheng Biotech Co. Ltd. All other chemicals were of analytical grade and used without further purification.

2.2. Sample Preparation. A bovine LF stock solution was made by dissolving the appropriate amount in amount of LF in $0.05 \mathrm{M}$ phosphate buffer ( $\mathrm{pH}$ 7.4). Solutions of Luteolin, Quercetin, and Naringenin were prepared daily by dissolving the appropriate amount in pure alcohol to obtain concentration $10 \mathrm{mM}$, respectively.

Samples were prepared by mixing BLF solutions and LTL, QCT, or NGN of varying proportions. The resulting ethanol concentration was approximately $1 \%$, which had no appreciable effect on protein structure. All samples were kept at $277 \mathrm{~K}$ before determination.

\subsection{Procedures}

2.3.1. Fluorescence Measurement. Fluorescence measurements were run with a spectrofluorometer, Model LS-55 (PerkinElmer, USA), equipped with a thermostatic sample compartment, and connected with a circulating bath (Lauda, K-2R; Brinkmann Instruments, Westbury, NY, USA).

$2.5 \mathrm{~mL}$ of $0.5 \mu \mathrm{M}$ BLF solution was put in $1.0 \mathrm{~cm}$ quartz cells which were titrated by successive additions of $2.5 \mathrm{mM}$ LTL, QCT, or NGN solutions according to the concentrations of $0,1,2,3,4,5,6,7$, and $8 \mu \mathrm{M}$, respectively. Fluorescence emission spectra of BLF were scanned from 300 to $500 \mathrm{~nm}$ with the excitation wavelength of $280 \mathrm{~nm}$ at $300 \mathrm{~K}, 305 \mathrm{~K}$, and $310 \mathrm{~K}$.

The band-widths of excitation and emission wavelength were set at $8 \mathrm{~nm}$, and the scanning speed was $220 \mathrm{~nm} / \mathrm{min}$. The synchronous fluorescence spectra were determined in the continuous range of 200 to $500 \mathrm{~nm}$ with $\Delta \lambda$ at 15 and $60 \mathrm{~nm}$.

In order to avoid the inner filter effects of protein and ligands, absorbance measurements were made at excitation and emission wavelengths of bovine LF. The fluorescence intensity was corrected using the following equation [11]:

$$
\mathrm{F}_{\text {cor }}=\mathrm{F}_{\text {obsd }} \mathrm{e} \frac{\mathrm{A}_{280}+\mathrm{A}_{345}}{2}
$$

where $\mathrm{F}_{\text {cor }}$ and $\mathrm{F}_{\text {obsd }}$ are the absorption-corrected fluorescence and the observed fluorescence, while $\mathrm{A}_{280}$ and $\mathrm{A}_{344}$ are the sums of the absorbance of protein and ligand at excitation and emission wavelengths, respectively. However, in the flavonoid-BLF ultraviolet absorption spectrum, LTL, QCT, or NGN does not exhibit any absorption at the excitation wavelength $(280 \mathrm{~nm})$ or at the emission wavelength $(345 \mathrm{~nm})$. So, no inner filter effects need to be considered [14].

\subsubsection{UV-Visible Absorption Measurement. Absorbance spec-} tra were recorded on a VARIAN 100 UV-VIS spectrophotometer (Varian Australia Pty. Ltd., Australia).

$2.5 \mathrm{~mL}$ of $1 \mu \mathrm{M}$ BLF solution in $1.0 \mathrm{~cm}$ quartz cells was titrated by successive additions of $2.5 \mathrm{mM}$ LTL, QCT, or NGN solutions for concentrations of $0,1,2,3,4,5,6,7$, and $8 \mu \mathrm{M}$, respectively, and their absorption spectra were recorded from 200 to $500 \mathrm{~nm}$.

2.4. Molecular Docking. SYBYL X-2.0 software was used for molecular docking based on its Surflex-Dock module. The crystal structure of protein with the resolution of 2.6 $\AA$ was downloaded from the Protein Data Bank (PDB ID: $1 \mathrm{M} 17)$. Protein was prepared using protein structure preparation module of the SYBYL X-2.0 software. All the water molecules and ligand were deleted, and hydrogen atoms were added to the crystal structure. In addition, the terminaltreatment of the protein was added charge. Small molecules were minimized at physiological $\mathrm{pH} 7.4$ with hydrogen atoms and charge by using Powell energy gradient method and the Gasteiger-Huckel system. The tautomeric form of the minimized inhibitor was free.

2.5. Statistical Analysis. All determinations were performed in triplicate and the mean values and standard deviations 


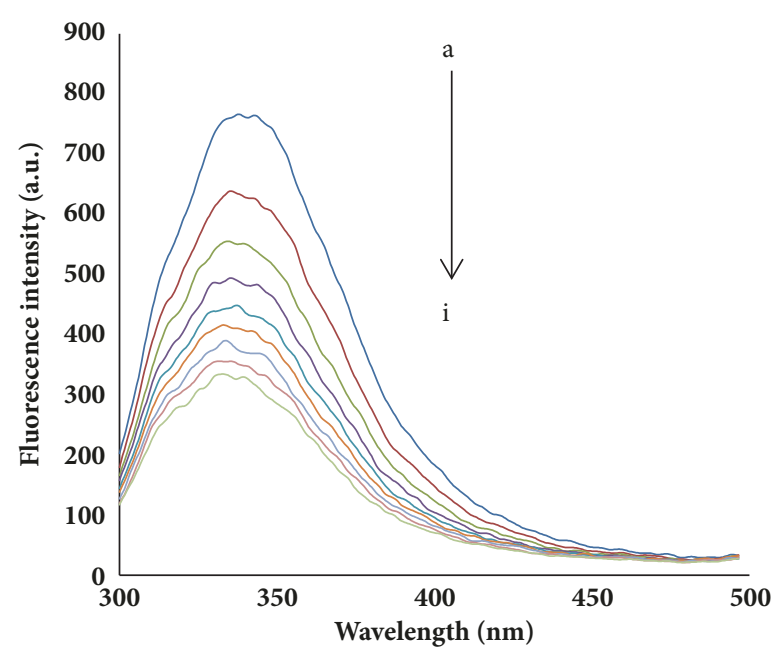

(a)

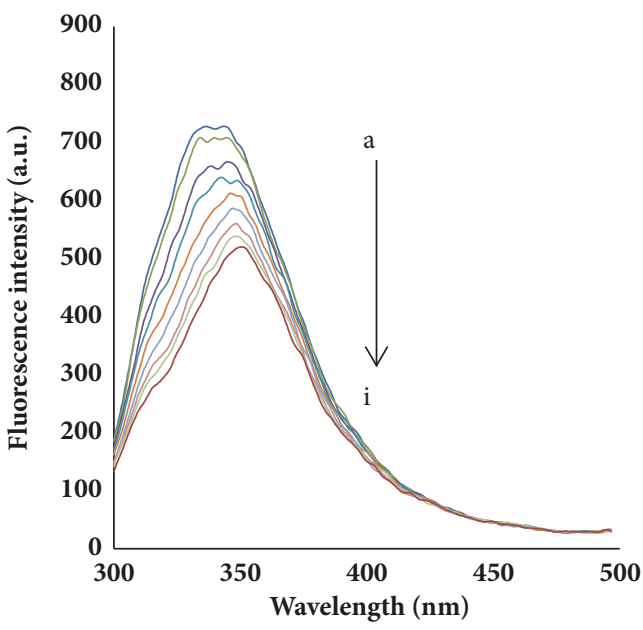

(c)

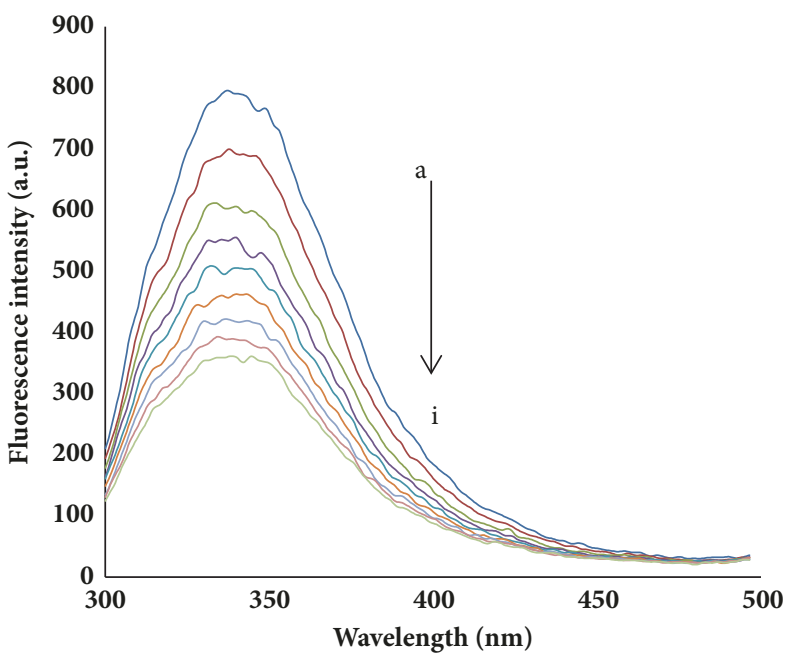

(b)

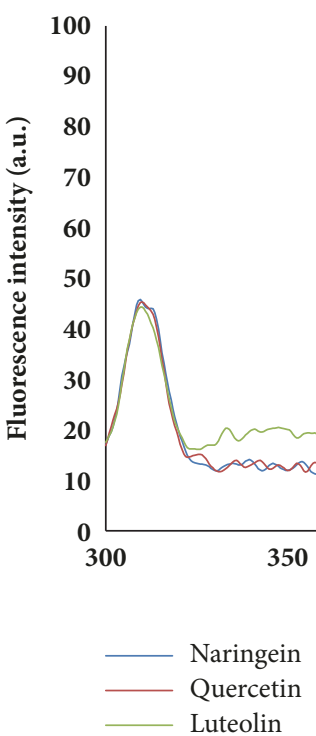

FIGURE 1: The fluorescence quenching spectra of BLF in the presence of different concentrations of three flavonoids. ((a) The fluorescence quenching spectra of BLF in the presence of different concentrations of LTL. (b) The fluorescence quenching spectra of BLF in the presence of different concentrations of QCT. (c) The fluorescence quenching spectra of BLF in the presence of different concentrations of NGN. $\mathrm{C}_{\mathrm{BLF}}=1.0$ $\times 10^{-6} \mathrm{~mol} / \mathrm{L}$, a-i: $\mathrm{C}_{\mathrm{LTL}}=\mathrm{C}_{\mathrm{QCN}}=\mathrm{C}_{\mathrm{NGN}}=(0,1,2,3,3,5,6,7,8) \times 10^{-6} \mathrm{~mol} \cdot \mathrm{L}^{-1}, \mathrm{~T}=300 \mathrm{~K}, \mathrm{pH}=7.4, \lambda \mathrm{ex}=280 \mathrm{~nm}$. $)$

were analyzed by using SPSS 13.0 for Windows (SPSS Inc., IL, USA).

\section{Results}

3.1. Interactions between Flavonoids and BLF. As shown in Figure 1, BLF's fluorescence intensity of approximately $344 \mathrm{~nm}$ regularly decreased with different concentrations of the added flavonoids. Furthermore, the maximum wavelength of BLF had a hypochromatic shift from 344 to $331 \mathrm{~nm}$, as shown in Figure 1(a), and from 344 to $338 \mathrm{~nm}$, as shown in Figure 1(b), respectively. It has been shown that Trp creates a more hydrophobic environment [21]. However, the peak position had a redshift from 344 to $349 \mathrm{~nm}$, as shown in
Figure 1(c), implying that the polarity of the microenvironment around Trp increased after NGN was added to the BLF solution. The fluorescence intensity of BLF decreased with increasing flavonoid concentrations, which indicated that the BLF conformation may be changed and that intermolecular energy transfer occurred between BLF and flavonoid [11].

The influence of the flavonoids on the UV-visible spectra of BLF is shown in Figure 2. BLF without addition exhibited a maximum absorption peak at $280 \mathrm{~nm}$ (curve j), but BLF showed a blueshift from $280 \mathrm{~nm}$ to $268 \mathrm{~nm}$ or from $280 \mathrm{~nm}$ to $270 \mathrm{~nm}$ with the addition of LTL or QCT, respectively, and a redshift from $280 \mathrm{~nm}$ to $283 \mathrm{~nm}$ with the addition of NGN (curves a-i). Simultaneously, the peak values gradually increased, suggesting the interactions between three 


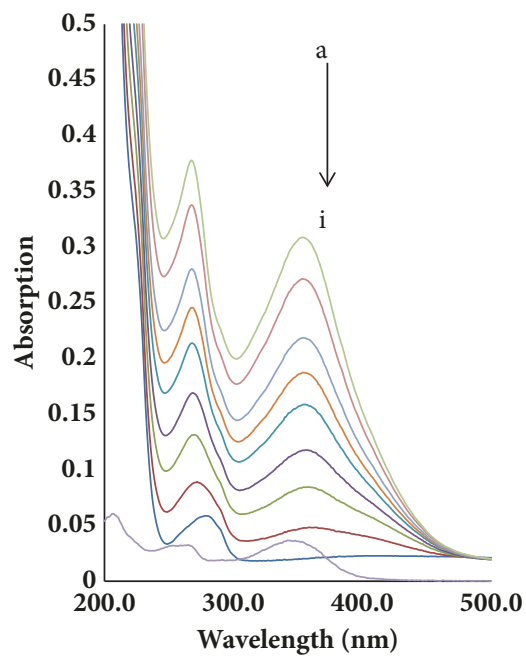

(a)

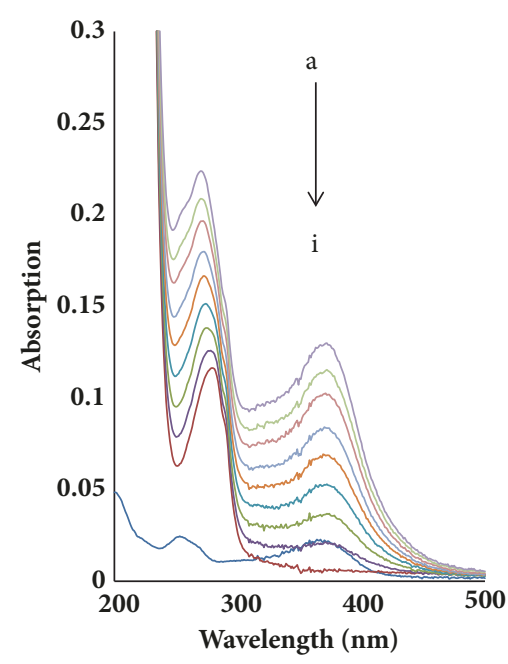

(b)

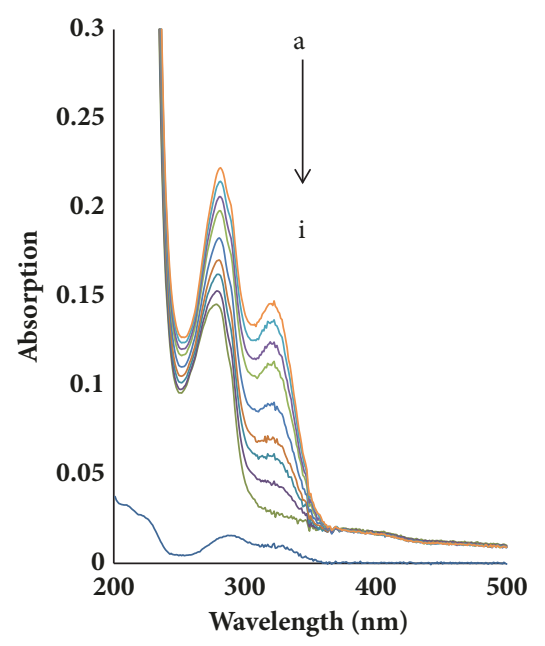

(c)

FIGURE 2: UV-visible spectra of BLF in the presence of three flavonoids. ((a) UV-visible spectra of BLF in the presence of LTL. (b) UV-visible spectra of BLF in the presence of QCT. (c) UV-visible spectra of BLF in the presence of NGN. $C_{B L F}=1.0 \times 10^{-6}$ mol/L, a-i: $C_{L T L}=C_{Q C N}=C_{N G N}$ $=(0,1,2,3,4,5,6,7,8) \times 10^{-6} \mathrm{~mol} / \mathrm{L} . \mathrm{j}: 8 \mu \mathrm{M}$ flavonoid alone. $)$

TABLE 1: Stern-Volmer equation of fluorescence quenching of BLF due to its interaction with LTL, QCT, or NGN and values of Ksv, Kq, and Runder temperatures of $300 \mathrm{~K}, 305 \mathrm{~K}$, and $310 \mathrm{~K}$. All titrations were performed in triplicate.

\begin{tabular}{lccccc}
\hline & $T(\mathrm{~K})$ & $K s v\left(\times 10^{4} \mathrm{M}^{-1}\right)$ & $K q\left(\times 10^{12} \mathrm{M}^{-1} \mathrm{~s}^{-1}\right)$ & $R^{\mathrm{a}}$ (correlation coefficien) & \multicolumn{1}{c}{$S D^{\mathrm{b}}$} \\
\hline \multirow{3}{*}{ Luteolin } & 300 & 10.08 & 10.08 & 0.997 & 0.999 \\
& 305 & 9.14 & 9.14 & 0.997 & 0.01584 \\
& 310 & 8.94 & 8.94 & 0.998 & 0.03678 \\
Quercetin & 300 & 9.81 & 9.81 & 0.998 & 0.997 \\
& 305 & 8.58 & 8.58 & 0.994 & 0.03384 \\
\multirow{3}{*}{ Naringein } & 310 & 3.00 & 8.00 & 0.997 & 0.06257 \\
& 300 & 3.14 & 3.67 & 0.999 & 0.04757 \\
\hline
\end{tabular}

${ }^{a} R$ is the correlation coefficient for the Ksv values.

${ }^{\mathrm{b}} \mathrm{SD}$ is the standard deviation for the Ksv values.

flavonoids and BLF occurred, primarily induced by hydrogen bonding or hydrophobic interaction. Moreover, the addition of the flavonoids resulted in the presence of a new peak at $370 \mathrm{~nm}$, which is the characteristic peak of flavonoids, and the flavonoids' absorption values increased with the increase in flavonoids concentration, which further confirmed the occurrence of interactions between three flavonoids and BLF.

3.2. The Fluorescence Quenching Mechanism. For experiments performed with large molar protein to drug ratios, it is hypothesized that each binding site is active in the binding drug, is identical, and acts independently. Given the validity of these assumptions, the fluorescence quenching behaviour can be analyzed using the Stern-Volmer and Lehrer equations for linear and nonlinear (hyperbolic) fits, respectively. Dynamic quenching was calculated according to Stern-Volmer equation [22]:

$$
\frac{F_{0}}{F}=1+K q \tau_{0}[Q]=1+K s v[Q]
$$

where $F_{0}$ and $F$ are the relative fluorescence intensities of BLF at $345 \mathrm{~nm}$ in the absence and presence of a quencher, $\mathrm{Kq}$ is the quenching rate constant of the bimolecule, $\tau_{0}$ is the average lifetime of biomolecule without active constituents, $[Q]$ is the concentration of active constituent, and $K_{S V}$ stands for the Stern-Volmer dynamic quenching constant.

To confirm the possible quenching mechanism of the three flavonoids binding to BLF, the dynamic quenching parameters, $K s v$ and $K q$, were achieved from the experimental results using Stern-Volmer equation. The fluorescence lifetime of the biopolymer is $10^{-8} \mathrm{~s}$. The linear plot of $\mathrm{F}_{0} / \mathrm{F}$ as a function of Quercetin concentration is given in Figure 3; the results are listed in Table 1. The maximum scatter collision quenching constant of various quenchers with the biopolymer is $2.00 \times 10^{10} \mathrm{M}^{-1}$ [23]. The values of 
TABLE 2: Binding constant $K_{a}$ and number of binding sites of the interaction of LTL, QCT, or NGN with BLF.

\begin{tabular}{|c|c|c|c|c|c|}
\hline & $T(\mathrm{~K})$ & $K a\left(\times 10^{4} \mathrm{M}^{-1}\right)$ & $n^{\mathrm{e}}$ & $R^{\mathrm{c}}$ & $S D^{\mathrm{d}}$ \\
\hline \multirow{3}{*}{ Luteolin } & 300 & 8.889 & 1.052 & 0.997 & 0.06442 \\
\hline & 305 & 8.410 & 1.020 & 0.999 & 0.03862 \\
\hline & 310 & 7.257 & 1.079 & 0.998 & 0.03582 \\
\hline \multirow{3}{*}{ Quercetin } & 300 & 9.689 & 1.034 & 0.998 & 0.04577 \\
\hline & 305 & 7.215 & 1.050 & 0.998 & 0.03874 \\
\hline & 310 & 5.347 & 1.105 & 0.997 & 0.05829 \\
\hline \multirow{3}{*}{ Naringein } & 300 & 3.05 & 1.076 & 0.999 & 0.04278 \\
\hline & 305 & 2.35 & 1.166 & 0.971 & 0.08442 \\
\hline & 310 & 1.79 & 1.078 & 0.992 & 0.06793 \\
\hline
\end{tabular}

${ }^{\mathrm{c}} R$ is the correlation coefficient for the $K_{a}$ values.

d $\mathrm{SD}$ is the standard deviation for the $K_{a}$ values.

e The binding site $(n)$ approximated to 1 .

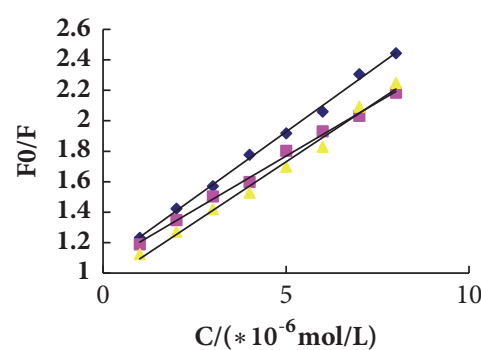

- $300 \mathrm{~K}$

- $305 \mathrm{~K}$

$\triangle 310 \mathrm{~K}$

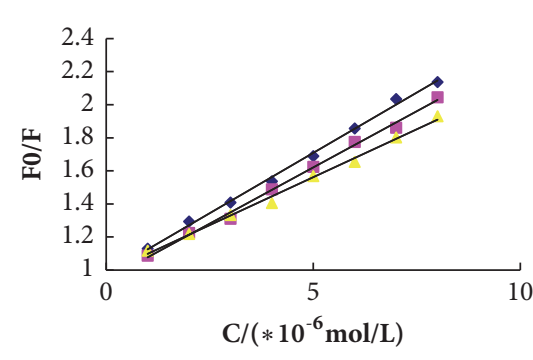

- $300 \mathrm{~K}$

- $305 \mathrm{~K}$

$\triangle 310 \mathrm{~K}$

(a)

(b) (c)

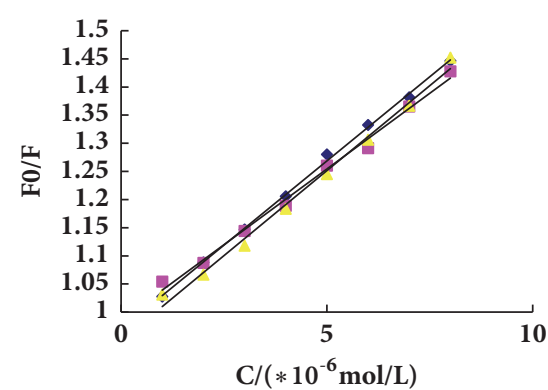

- $300 \mathrm{~K}$

- $305 \mathrm{~K}$

$+310 \mathrm{~K}$

FIGURE 3: Stem-Volmer plots of three flavonoids-BLF systems at different temperatures. ((a) Stem-Volmer plots of LTL-BLF systems at different temperatures. (b) Stem-Volmer plots of QCT-BLF systems at different temperatures. (c) Stem-Volmer plots of NGN-BLF systems at different temperatures.)

$K q$ decreased with increased temperature and were greater than the limiting diffusion constant, which suggested that the possible quenching mechanism was a static quenching process accompanied with the formation of BLF-flavonoid complexes, and dynamic collision was negligible.

3.3. Binding Parameters. For the static quenching, the binding constant $\left(K_{a}\right)$ and the number of binding sites $(n)$ can be calculated using the following equation [24]:

$$
\lg \left[\frac{\left(F_{0}-F\right)}{F}\right]=\lg K_{a}+n \lg [Q]
$$

And, for $\mathrm{n} \approx 1,(3)$ can be rewritten as follows [25]:

$$
\frac{F 0}{(F 0-F)}=1+K_{a}^{-1}[Q]^{-1}
$$

The results are exhibited in Tables 1 and 2.

BLF interacts with LTL, QCT, and GNG to form 1:1 complexes. With increased temperature, the values of $K_{a}$ obtained at the excitation wavelength of $280 \mathrm{~nm}$ decreased, which may indicate the formation of an unstable compound.
The unstable compound would be partly decomposed with the increased temperature. Moreover, it has been reported that an equilibrium between monomeric and associated states may exist in solution due to self-association at high concentrations [26].

The binding constants have an order of magnitude of $10^{4}$ $\mathrm{L} \cdot \mathrm{mol}^{-1}$, which means that the binding strength is relatively high, indicating strong binding affinities. This is further evidence that the binding interaction between flavonoids and bovine lactoferrin occurs. The binding constants obtained for the BLF complexes are in the range of $1.79 \times 10^{4} \mathrm{M}^{-1}-9.68 \times$ $10^{4} \mathrm{M}^{-1}$, and similar results were generally observed for BLartemisinin complexes $\left(1.6 \times 10^{4} \mathrm{M}^{-1}-6.2 \times 10^{4} \mathrm{M}^{-1}\right)$ [14]. These results indicated that LTL and QCN bound to HSA to a larger extent than to NGN, which signifies that the affinity of BLF to LTL or QCN was higher than that between BLF and QCN.

3.4. Thermodynamic Parameter and Nature of the Binding Force. There are some interaction forces between an active constituent and a biomacromolecule, such as hydrophobic forces, van der Waals interactions, electrostatic interactions, 


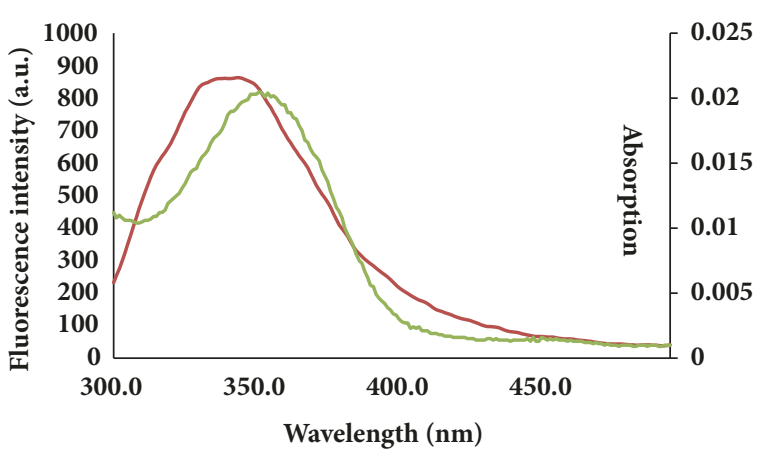

(a)

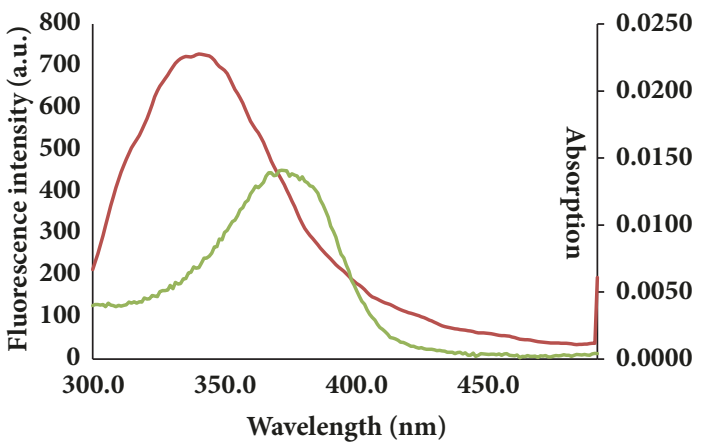

(b)

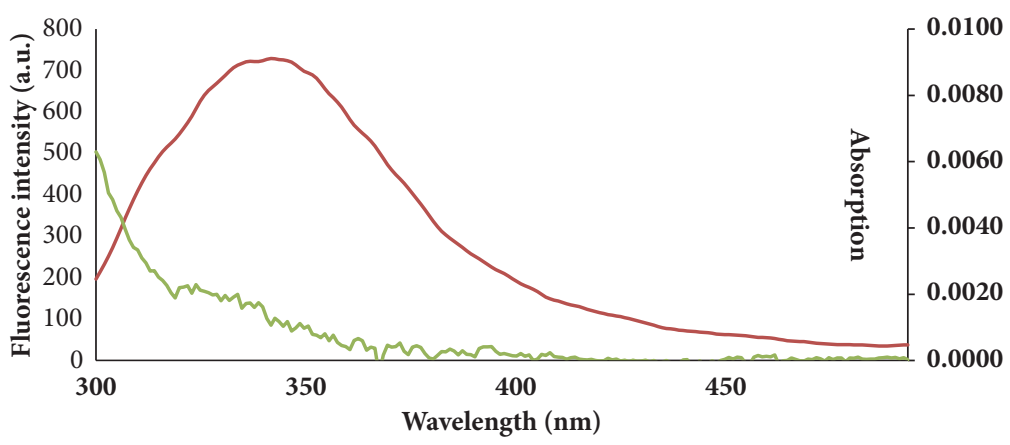

(c)

FIGURE 4: Overlap of absorption spectra of three flavonoids and fluorescence mission spectra of BLF. (a) Overlap of absorption spectra of LTL. (b) Overlap of absorption spectra of QCT. (c) Overlap of absorption spectra of NGN. $C_{B L F}=C_{\text {LTL }}=C_{Q C N}=C_{\mathrm{NGN}}=1.0 \times 10^{-6} \mathrm{~mol} \cdot \mathrm{L}^{-1}$.

TABLE 3: Thermodynamic parameters of the interaction of LTL, QCT, or NGN with BLF.

\begin{tabular}{lcccc}
\hline & $K_{a} \times 10^{4}$ at $310 \mathrm{~K}\left(\mathrm{M}^{-1}\right)$ & $\left.\triangle H(\mathrm{~kJ} \mathrm{~mol})^{-1}\right)$ & $\triangle S\left(\mathrm{~J} \mathrm{~mol}^{-1} \mathrm{~K}^{-1}\right)$ & $\triangle G$ at $\left.310 \mathrm{~K}(\mathrm{~kJ} \mathrm{~mol})^{-1}\right)$ \\
\hline Luteolin & 7.257 & -15.30 & +41.79 & -27.84 \\
Quercetin & 5.347 & -31.48 & -10.13 & -28.42 \\
Naringenin & 1.79 & 3.57 & 74.60 & -26.86 \\
\hline
\end{tabular}

and hydrogen bonds. The signs and magnitudes of thermodynamic parameters that are calculated from the van 't Hoff equation account for the main forces maintaining protein stability [27]. From Table 3, it is observed that the negative sign for $\triangle G$ indicates the binding spontaneity of the three flavonoids with BLF (see (5)-(6)). According to the views of Timasheff, Ross, and Subramanian, from the model of the interaction between the drug and HSA, the interactions can be concluded: (1) $\triangle H>0$ and $\triangle S>0$, hydrophobic forces; (2) $\triangle H<0$ and $\triangle S<0$, van der Waals interactions and hydrogen bonds; and (3) $\triangle H<0$ and $\triangle S>0$, electrostatic interactions. Hence, the results showed that the main force between QCT and LTL with BLF is van der Waals interactions and hydrogen bonds, and electrostatic interactions played a major role in the binding process between BLF and NGN.

$$
\begin{aligned}
\ln K_{a} & =-\frac{\triangle H}{R T}+\frac{\triangle S}{R} \\
\triangle G & =\triangle H-T \triangle S
\end{aligned}
$$

3.5. Energy Transfer from BLF and Quercetin. According to Forster's nonradiative energy transfer theory [28], the energy can be transferred from the donor to the acceptor when a donor is emitted by fluorescence.

The fluorescence quenching of BLF after binding to Quercetin indicated that the transfer of energy between Quercetin and BLF occurred. Spectroscopy of the donor is malformed due to this interaction. The related parameters, including energy transfer efficiency $E$, the distance $(r)$, and the critical energy transfer distance $\left(R_{0}\right)$, are calculated by the following equations:

$$
\begin{aligned}
E & =\frac{R_{0}^{6}}{\left(R_{0}^{6}+r_{0}^{6}\right)} \\
E & =1-\frac{F}{F_{0}} \\
R_{0}^{6} & =8.8 \times 10^{-25} K^{2} N^{-4} \Phi J \\
J & =\frac{\sum\left(\mathrm{F}(\lambda) \cdot \varepsilon(\lambda) \cdot \lambda^{4} \cdot \Delta \lambda\right)}{\sum(\mathrm{F}(\lambda) \cdot \Delta \lambda)}
\end{aligned}
$$


TABLE 4: Binding distances of the interaction of BLF with LTL, QCT, or NGN.

\begin{tabular}{lcccc}
\hline compound & $\mathrm{J}\left(\times 10^{-17} \mathrm{~cm}^{3} \mathrm{lmol}^{-1}\right)$ & $\mathrm{R}_{0}(\mathrm{~nm})$ & $\mathrm{E}$ & $\mathrm{R}(\mathrm{nm})$ \\
\hline Quercetin & 5.45 & 2.59 & 0.112 & 3.66 \\
Naringenin & 3.61 & 2.11 & 0.042 & 3.56 \\
Luteolin & 6.36 & 2.80 & 0.034 & 4.89 \\
\hline
\end{tabular}

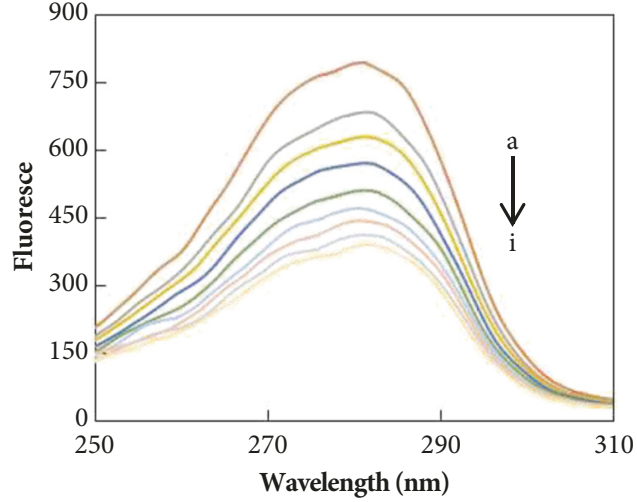

(a)

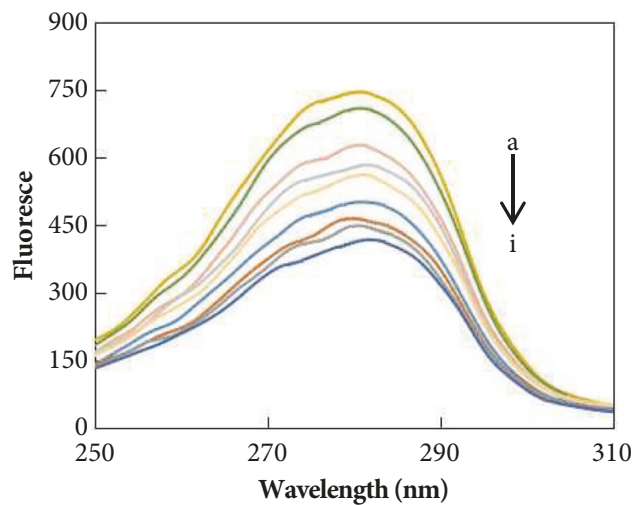

(c)

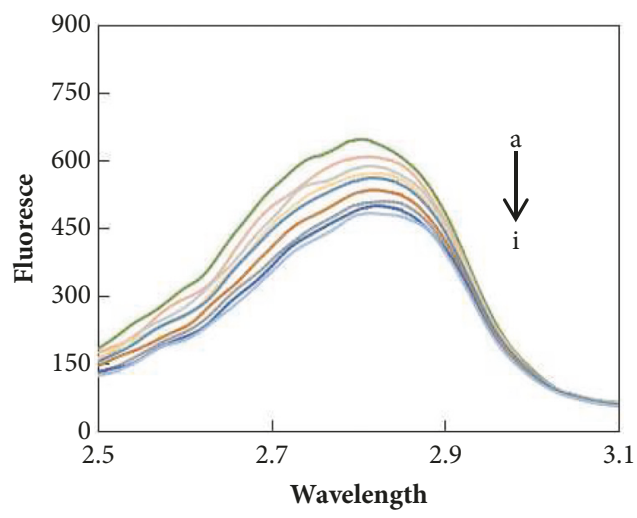

(e)

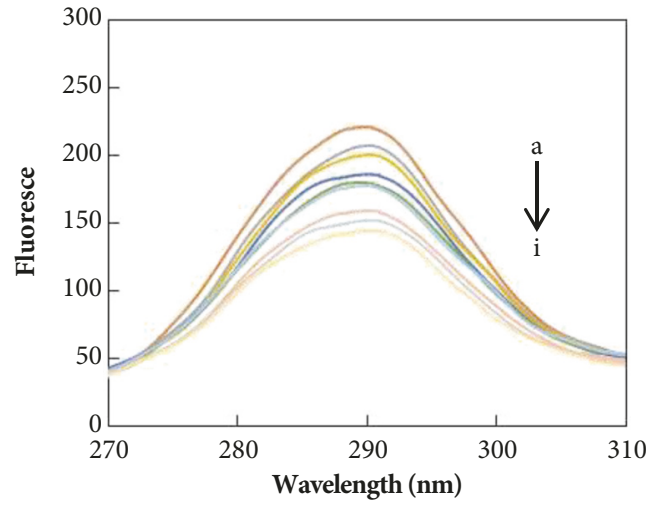

(b)

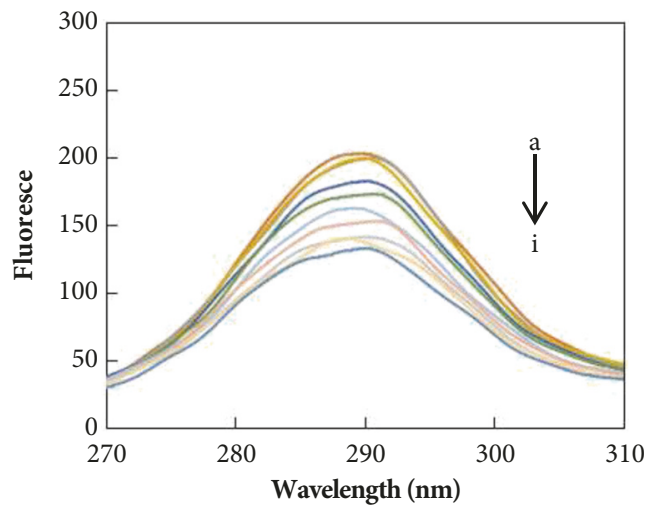

(d)

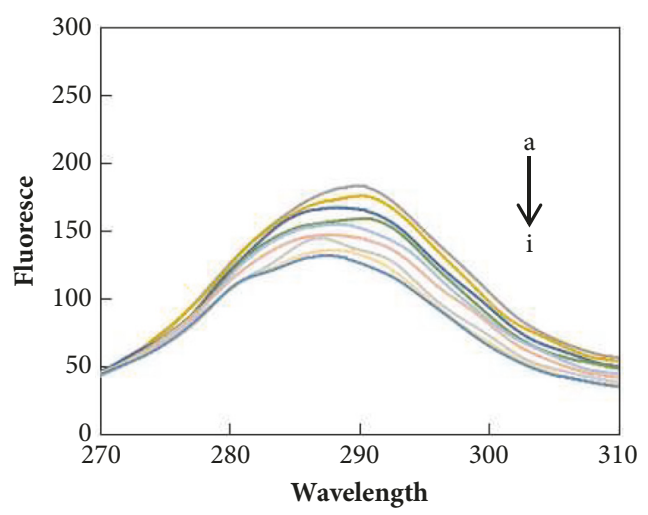

(f)

FIGURE 5: Effect of the drug on the synchronous fluorescence spectrum of BLF. (a) $\Delta \lambda=15 \mathrm{~nm}$ UA-BLF; (b) $\Delta \lambda=60 \mathrm{~nm}$ OA-BFL; (c) $\Delta \lambda=15 \mathrm{~nm}$ UA-BFL; (d) $\Delta \lambda=60 \mathrm{~nm} \mathrm{OA-BFL;} \mathrm{(e)} \Delta \lambda=15 \mathrm{~nm}$ UA-BFL; (f) $\Delta \lambda=60 \mathrm{~nm} \mathrm{OA-BFL.} \mathrm{C}_{\mathrm{BLF}}=1 \mu \mathrm{M}$, a-i: $\mathrm{C}_{\mathrm{LTL}}=\mathrm{C}_{\mathrm{QCN}}=\mathrm{C}_{\mathrm{NGN}}$ $=(0,1,2,3,3,5,6,7,8) \times 10^{-6} \mathrm{~mol} \cdot \mathrm{L}^{-1}$. 


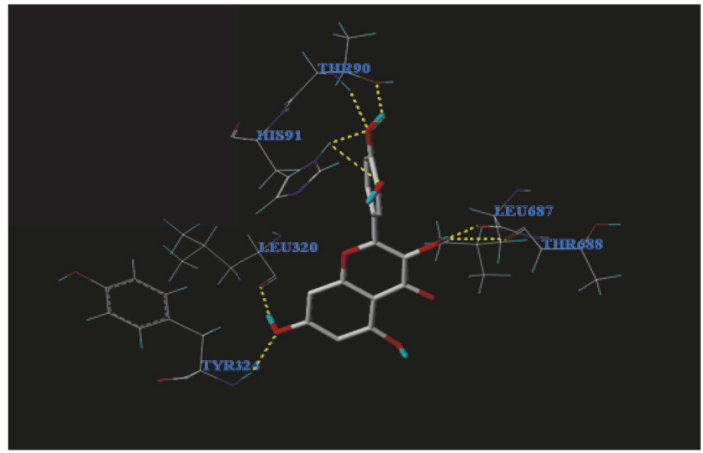

(a)

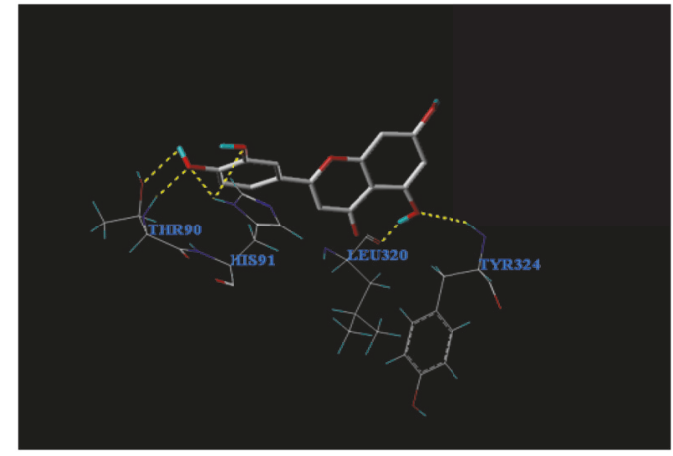

(b)

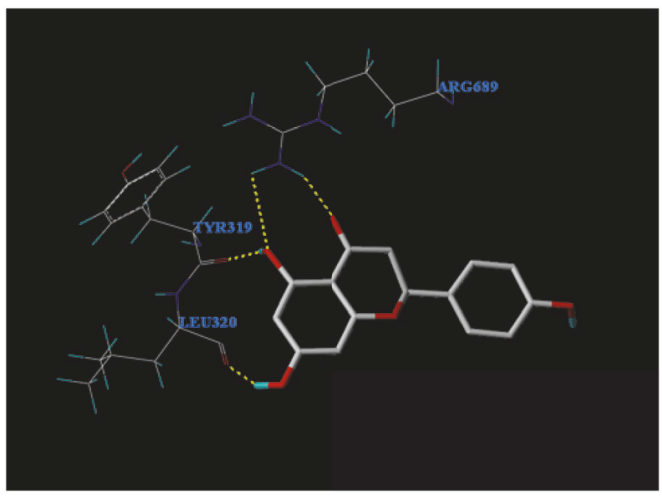

(c)

FIGURE 6: Molecule docking. (Molecule docking between LTL and BLF. Molecule docking between QCT and BLF. Molecule docking between NGN and BLF.)

where $R_{0}$ is the critical distance when the transfer efficiency is $50 \%, K^{2}$ stands for the spatial orientation factor of the dipole, $N$ is the refractive index of the medium, $F$ stands for the fluorescence quantum yield of the donor, and $J$ stands for the overlap integral of the fluorescence emission spectrum of the donor and the absorption spectrum of the acceptor. $F(\lambda)$ stands for the fluorescence intensity of the donor and $\varepsilon(\lambda)$ is the molar absorptivity of the acceptor when the wavelength is $\lambda$. In the experiment, $K^{2}$ is $2 / 3, N$ is 1.36 , and $\Phi$ is supposed to be the same as the fluorescence quantum yield of tryptophan (0.15). The concentration of flavonoid is very low, so its absorbance values are low. The calculated results are shown in Figure 4 and Table 4 . The distance (r) between Quercetin and BLF was less than $7 \mathrm{~nm}$, which verified the presence of nonradiation energy transfer between Quercetin and BLF [29].

3.6. Effect of Flavonoids on BLF Conformation. Synchronous fluorescence of the flavonoid-BLF systems was detected to investigate the microenvironment change of amino acid residues, which is related to a shift in the maximum emission wavelength, and the results are shown in Figure 5. When $\Delta \lambda$ ( $\Delta \lambda=\lambda$ emission- $\lambda$ excitation) is 15 or $60 \mathrm{~nm}$, the synchronous fluorescence provides the characteristic information of tyrosine residues or tryptophan residues, respectively, due to the changes of the polarity in the microenvironment [30].
Obviously, the conformations of BLF were changed with the addition of LTL, QCT, and NGN. A redshift effect suggests that the polarity around the tyrosine residues increased, and the hydrophobicity decreased; a hypochromatic shift effect suggests that the polarity decreased around the tryptophan residues and increased in the hydrophobicity.

3.7. Analysis of Molecular Docking. Molecular docking of the three flavonoids with BLF was studied using SYBYL$\mathrm{X}-2.0$. Figure 6 shows that Luteolin (LTL) and Quercetin (QCT) bind to BLF via hydrogen bonds. LTL interacts with THR90 and HIS91 with two hydrogen bonds, respectively; and it also binds to BLF at LEU320, TYR324, LEU687, and THR688 with one hydrogen bond, respectively. In this study, there are a total of eight hydrogen bonds when LTL interacts with BLF. QCT forms two hydrogen bonds with THR90 and HIS91, respectively. In addition, QCT binds to BLF at LEU320 and TYR324 with one hydrogen bond, respectively. Hence, it is suggested that hydrogen bonds contribute the most to LTL and QCT interaction with BLF. NGN formed two hydrogen bonds with ARG689 and formed a single hydrogen bond with TYR319 and LEU320, respectively. Obviously, it is possible that other possible forces, such as hydrophobic interactions, are essential for the binding between NGN and BLF. The flavone nucleus is the main hydrophobic group, which interacts with BLF via a hydrophobic force. 
The docking results agreed well with the aforementioned spectroscopy results.

\section{Conclusion}

In this work, we studied the interactions between BLF and 3 flavonoid compounds using the spectroscopy methods. The results revealed that LTL, QCT, and NGN interact with BLF to form new complexes, which leads to the static quenching of the fluorescence of BLF and nonradiation energy transfer. In these results, both van der Waals interactions and hydrogen bonds played key roles in the binding process of Lut and Que with BLF; however, the main forces between BLF and NGN are electrostatic interactions. The binding affinity of the flavonoids to BLF was the greatest for LTL followed by QCT and was least for NGN. The results of the UV-vis spectra and the synchronous fluorescence analysis showed that the formation of flavonoid-BLF complexes induced changes of different degrees in the protein structure.

The above studies showed that the following relationships may exist for the structure and binding interactions of the three flavonoids with BLF: (1) hydroxyl groups at the 5,7 position of ring $A$ in the three flavonoids were key to determining the groups, (2) the increase of phenolic hydroxyls in the $\mathrm{B}$ ring of the flavonoids was favourable for drug binding to HSA, and (3) C4'-OH enhanced the binding affinity obviously, but $\mathrm{C} 3-\mathrm{OH}$ weakened the affinity.

The binding properties of flavonoids with proteins can help to characterize the biological process of the compound. The results of ligand protein binding can provide important guides for compound quantity (e.g., medicine dosage) and the design of new compounds (or drugs).

\section{Data Availability}

The data used to support the findings of this study are available from the corresponding author upon request.

\section{Conflicts of Interest}

The authors declare that there are no conflicts of interest regarding the publication of this paper.

\section{Authors' Contributions}

Junyi Huang, Zhenmin Liu, and Qiaorong Ma contributed equally to this work.

\section{Acknowledgments}

This work was supported by the Open Project Program of State Key Laboratory of Dairy Biotechnology, Bright Dairy \& Food Co. Ltd. (no. SKLDB2016-004), the National Key Technology R\&D Program of China (no. 2015BAD16B02), and the Shanghai Municipal Education Commission (no. 11YZ18).

\section{References}

[1] T. P. Cushnie and A. J. Lamb, "Antimicrobial activity of flavonoids," International Journal of Antimicrobial Agents, vol. 26, no. 5, pp. 343-356, 2005.

[2] T. P. T. Cushnie and A. J. Lamb, "Recent advances in understanding the antibacterial properties of flavonoids," International Journal of Antimicrobial Agents, vol. 38, no. 2, pp. 99-107, 2011.

[3] Edewor-Kuponiyi and T. Ibibia, "A review on antimicrobial and other beneficial effects of flavonoids," International Journal of Pharmaceutical Sciences Review and Research, vol. 21, no. 1, pp. 20-33, 2013.

[4] L. Adlerova, A. Bartoskova, and M. Faldyna, "Lactoferrin: a review," Veterinarni Medicina, vol. 53, no. 9, pp. 457-468, 2008.

[5] J. Chamani, N. Tafrishi, and M. Momen-Heravi, "Characterization of the interaction between human lactoferrin and lomefloxacin at physiological condition: Multi-spectroscopic and modeling description," Journal of Luminescence, vol. 130, no. 7, pp. 1160-1168, 2010.

[6] M. Guo, L.-Y. Zhang, W.-J. Lü, and H.-R. Cao, "Analysis of the spectroscopic characteristics on the binding interaction between tosufloxacin and bovine lactoferrin," Journal of Luminescence, vol. 131, no. 4, pp. 768-775, 2011.

[7] B. Fang, M. Zhang, M. Tian, L. Jiang, H. Y. Guo, and F. Z. Ren, "Bovine lactoferrin binds oleic acid to form an anti-tumor complex similar to HAMLET," Biochimica et Biophysica Acta (BBA) - Molecular and Cell Biology of Lipids, vol. 1841, no. 4, pp. 535-543, 2014.

[8] K. V. P. C. Mohan, P. Gunasekaran, E. Varalakshmi, Y. Hara, and S. Nagini, "In vitro evaluation of the anticancer effect of lactoferrin and tea polyphenol combination on oral carcinoma cells," Cell Biology International, vol. 31, no. 6, pp. 599-608, 2007.

[9] Z. Omidvar, A. Asoodeh, and J. Chamani, "Studies on the antagonistic behavior between cyclophosphamide hydrochloride and aspirin with human serum albumin: Time-resolved fluorescence spectroscopy and isothermal titration calorimetry," Journal of Solution Chemistry, vol. 42, no. 5, pp. 1005-1017, 2013.

[10] K. Vuignier, J. Schappler, J.-L. Veuthey, P.-A. Carrupt, and S. Martel, "Drug-protein binding: A critical review of analytical tools," Analytical and Bioanalytical Chemistry, vol. 398, no. 1, pp. 53-66, 2010.

[11] S. Marouzi, A. Sharifi Rad, S. Beigoli, P. Teimoori Baghaee, R. Assaran Darban, and J. Chamani, "Study on effect of lomefloxacin on human holo-transferrin in the presence of essential and nonessential amino acids: Spectroscopic and molecular modeling approaches," International Journal of Biological Macromolecules, vol. 97, pp. 688-699, 2017.

[12] S. H.-A. Tousi, M. R. Saberi, and J. Chamani, "Comparing the interaction of cyclophosphamide monohydrate to human serum albumin as opposed to holo-transferrin by spectroscopic and molecular modeling methods: Evidence for allocating the binding site," Protein and Peptide Letters, vol. 17, no. 12, pp. 15241535, 2010.

[13] S. Rashidipour, S. Naeeminejad, and J. Chamani, "Study of the interaction between DNP and DIDS with human hemoglobin as binary and ternary systems: Spectroscopic and molecular modeling investigation," Journal of Biomolecular Structure and Dynamics, vol. 34, no. 1, pp. 57-77, 2016.

[14] A. Veerappan, T. Eichhorn, M. Zeino, T. Efferth, and D. Schneider, "Differential interactions of the broad spectrum drugs 
artemisinin, dihydroartemisinin and artesunate with serum albumin," Phytomedicine, vol. 20, no. 11, pp. 969-974, 2013.

[15] M. Guo, X. Lu, Y. Wang, and P. E. Brodelius, "Comparison of the interaction between lactoferrin and isomeric drugs," Spectrochimica Acta Part A: Molecular and Biomolecular Spectroscopy, vol. 173, pp. 593-607, 2017.

[16] X. Wang, X. Y. Guo, L. Xu, B. Liu, L. L. Zhou, and X. F. Wang, "Studies on the competitive binding of cleviprex and flavonoids to plasma protein by multi-spectroscopic methods: a prediction of food-drug interaction," of Photochemistry Photobiology B Biology, pp. 192-199, 2017.

[17] W. Yang, F. Liu, C. Xu, F. Yuan, and Y. Gao, "Molecular interaction between (-)-epigallocatechin-3-gallate and bovine lactoferrin using multi-spectroscopic method and isothermal titration calorimetry," Food Research International, vol. 64, pp. 141-149, 2014.

[18] A. A. Moosavi-Movahedi, J. Chamani, H. Ghourchian, C. M. Sorenson, and N. Sheibani, "Electrochemical evidence for the molten globule states of cytochrome $\mathrm{c}$ induced by $\mathrm{N}$-alkyl sulfates at low concentrations," The Protein Journal, vol. 22, no. 1, pp. 23-30, 2003.

[19] J. Zhang, X.-F. Dai, and J.-Y. Huang, "Resveratrol Binding to Fibrinogen and its Biological Implication," Food Biophysics, vol. 7, no. 1, pp. 35-42, 2012

[20] Y. Li, F. Gao, F. Gao, F. Shan, J. Bian, and C. Zhao, "Study on the interaction between 3 flavonoid compounds and $\alpha$-amylase by fluorescence spectroscopy and enzymatic kinetics," Journal of Food Science, vol. 74, no. 3, pp. C199-C203, 2009.

[21] S. Y. Bi LLY and B. B. Wang, "Spectroscopic and voltammetric characterization of the interaction of two local anesthetics with bovine serum albumin," J Lumin, vol. 131, pp. 866-873, 2011.

[22] J. R. Lakowicz, Fluorescence Quenching: Theory and Applications, Principles of Fluorescence Spectroscopy, Kluwer Academic/Plenum Publishers, NY, USA, 1999.

[23] G. Roderic Eckenhoff, "Molecular interactions between inhaled anesthetics and proteins," Pharmacol Rev, pp. 343-368, 1997.

[24] M. Jiang, X. M. Xie, D. Zheng, Y. Liu, Y. X. Li, and X. J. Chen, "Spectroscopic studies on the interaction of cinnamic acid and its hydroxyl derivatives with human serum albumin," Journal of Molecular Structure, vol. 692, pp. 71-80, 2004.

[25] M.-X. Xie, X.-Y. Xu, and Y.-D. Wang, "Interaction between hesperetin and human serum albumin revealed by spectroscopic methods," Biochimica et Biophysica Acta (BBA) - General Subjects, vol. 1724, no. 1-2, pp. 215-224, 2005.

[26] L. Liang, H. A. Tajmir-Riahi, and M. Subirade, "Interaction of $\beta$ Lactoglobulin with resveratrol and its biological implications," Biomacromolecules, vol. 9, no. 1, pp. 50-56, 2008.

[27] D. ROSS AA, Themodynamics of protein in association reaction force contribution to stability, 1981.

[28] T. Föster OS, Modern quantum chemistry, vol. 3, 1996.

[29] I. Matei and M. Hillebrand, "Interaction of kaempferol with human serum albumin: A fluorescence and circular dichroism study," Journal of Pharmaceutical and Biomedical Analysis, vol. 51, no. 3, pp. 768-773, 2010.

[30] P. R. Callis, "La and Lb transitions of tryptophan: applications of theory and experimental observation to fluorescence of proteins," Methods in Enzymology, vol. 237, pp. 113-150, 1997. 


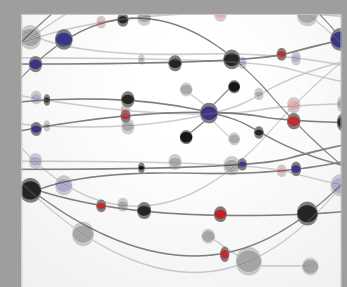

The Scientific World Journal
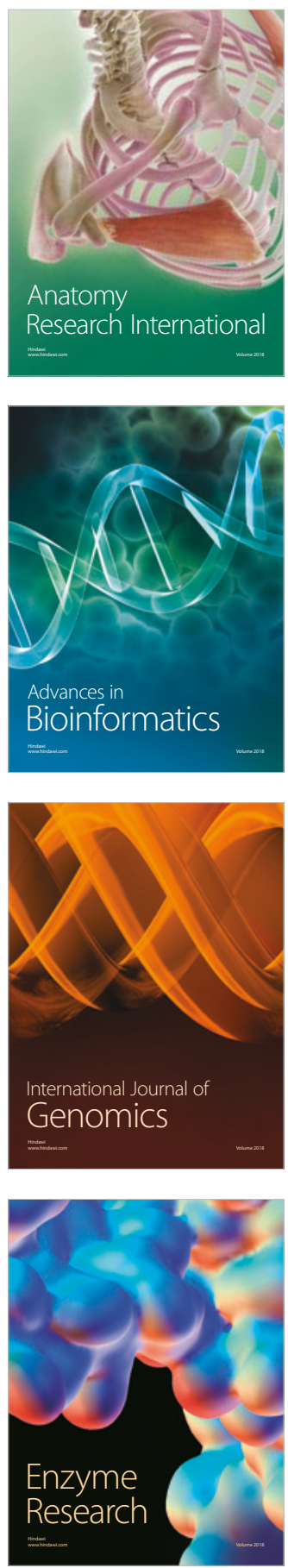
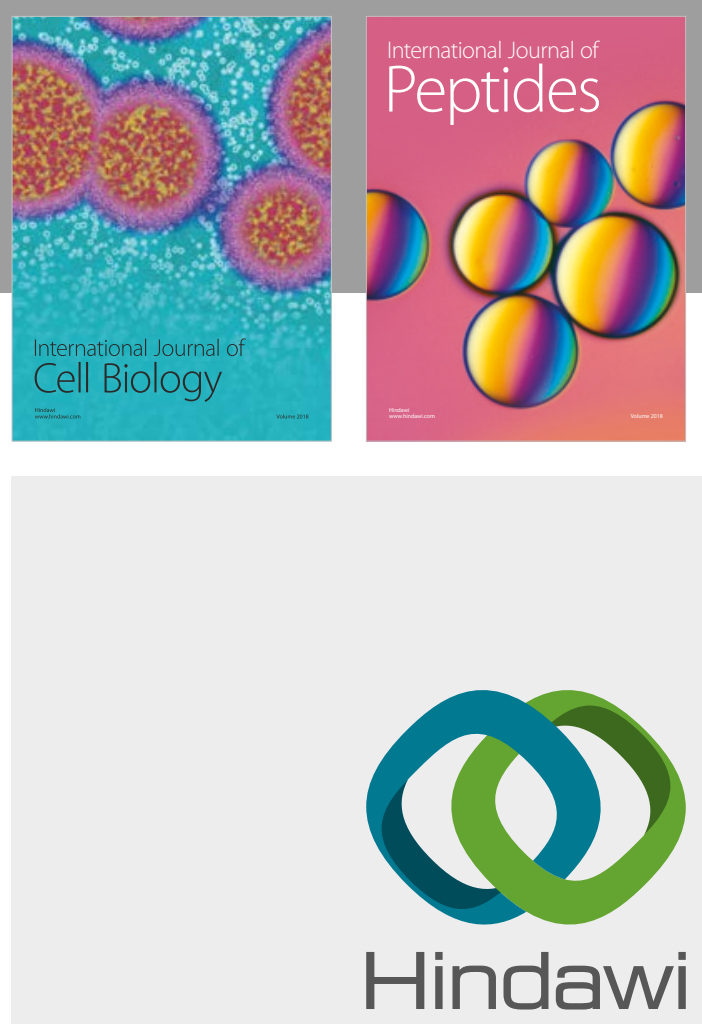

Submit your manuscripts at

www.hindawi.com
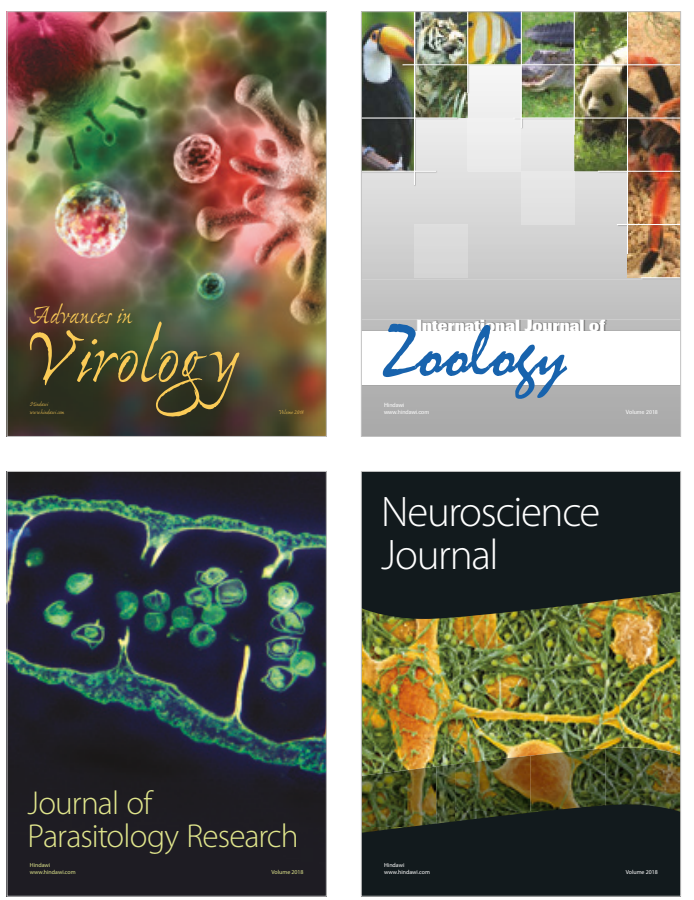
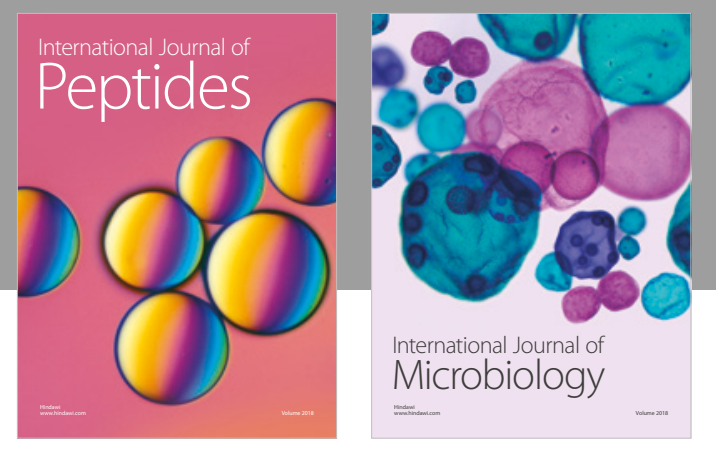

nternational Journal of Microbiology
Journal of
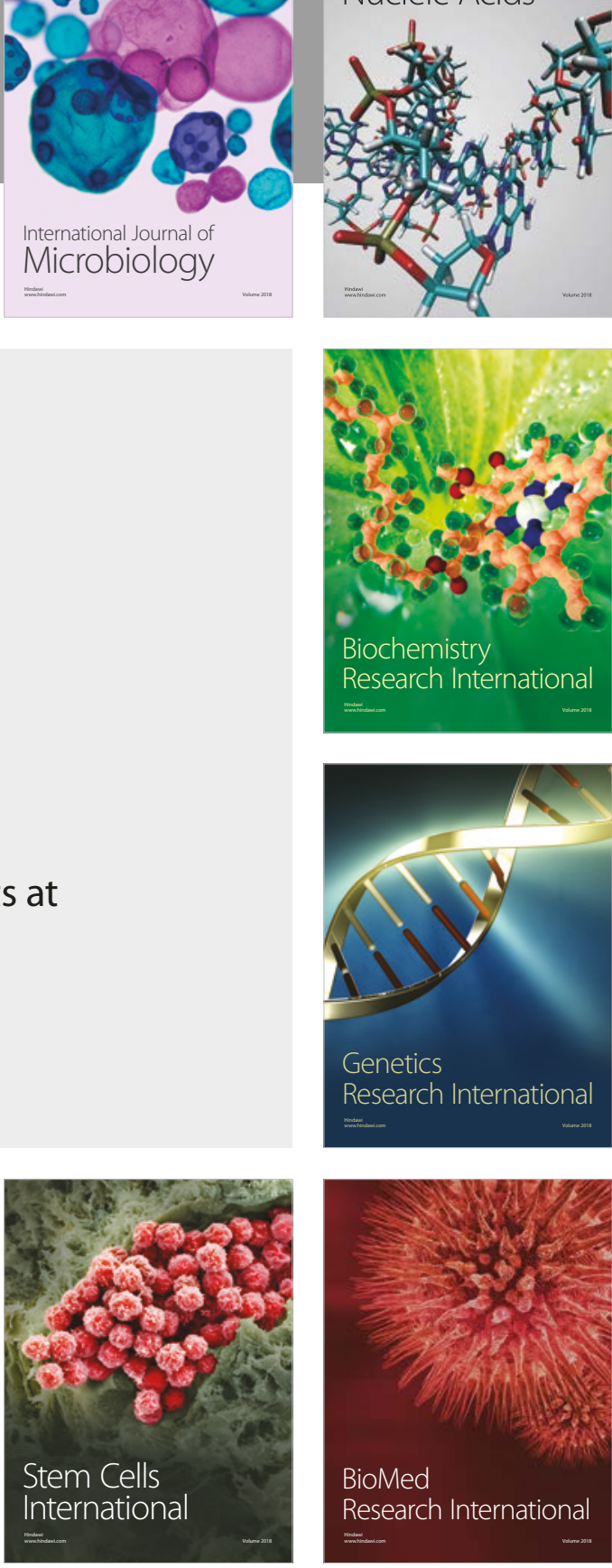
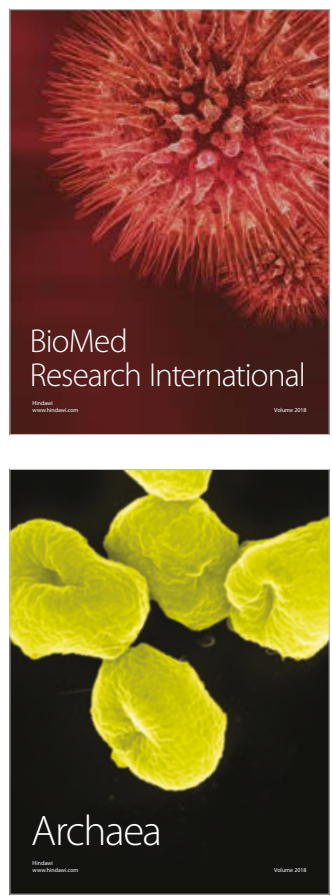